\title{
Structural Differences in the Umbilical Vein Wall after Full-Term and Pre-term Delivery
}

\author{
M. Bagyánszki ${ }^{1}$, Z. Novák ${ }^{2}$, N. Bódi ${ }^{1}$, H. Orvos ${ }^{3}$, A. PÁl $^{3}$ and É. Fekete ${ }^{1}$ \\ Addresses of authors: ${ }^{1}$ Department of Physiology, Anatomy and Neuroscience, ${ }^{2}$ Department of Paediatrics; ${ }^{3}$ Department \\ of Obstetrics and Gynaecology, University of Szeged, H-6726 Szeged, Közép fasor 52., Hungary; *Corresponding author: \\ Tel.: + (36) 62 544123; fax: + (36) 62 544291; e-mail: bmarcsi@ bio.u-szeged.hu
}

With 6 figures

Received May 2009; accepted for publication July 2009

\begin{abstract}
Summary
With the exception of its most proximal segment, the human umbilical cord lacks innervation. It might be expected, therefore, that a paracrine effect through the direct contact between the smooth muscle cells and the endothelium may be particularly important in the control of the fetoplacental circulation. In this study, electron microscopy and immunohistochemistry were applied to examine umbilical veins immediately after full-term and pre-term delivery. The smooth muscle cells in the upper layer of the tunica media exhibited long, foot-like processes with c-kit immunoreactivity. In the umbilical vein of full-term neonates more than $50 \%$ of these cell processes display a normal ultrastructure and they were closely associated with the lamina elastica interna. Whereas in pre-term infants more than $60 \%$ of these cell processes exhibit signs of severe shrinkage and detachedness from the lamina elastica interna. At the same time, the high level of immunoreactivity of the endothelial cells as regards the proapoptotic gene product Bax in preterm infants is indicative of an enhanced apoptotic process in these cells.
\end{abstract}

\section{Introduction}

The physiological uterine environment produces a continuous weight-related decrease of the umbilical blood flow (UmbBF), which results in a reduction of the fetal $\mathrm{O}_{2}$ transport (Dawes, 1969) in the final weeks of pregnancy. As a compensatory mechanism, with a normal vasodilator system in the umbilical vasculature (Sgambati et al., 2004), the total UmbBF increases during the last week of gestation (Link et al., 2007). We presume, therefore, that pathological changes in the structure of the umbilical vessels may interfere with this compensatory mechanism, leading to a severe reduction in placental perfusion, followed by pre-term delivery.

The UmbBF is significantly reduced in fetuses with restricted intrauterine growth (Ferrazzi et al., 2000), while poor correlations have been reported between the umbilical artery Doppler parameters and fetal growth retardation (Di Naro et al., 2002). These observations suggest that the UmbBF might provide a better index of placental perfusion than the umbilical artery velocity ratio. In the present work, therefore, we investigated the ultrastructure and immunohistochemical features of the umbilical vein wall in a search for structural elements that determine the maintenance of physiological placental perfusion until delivery.

With the exception of its most proximal segment, the human umbilical cord lacks innervation (Reilly and Russell, 1977; Fox and Khong, 1990). It might be expected, therefore, that a non-neuronal control of the fetoplacental circulation may be particularly important in these vessels. The importance of blood vessel endothelium in the control of vascular tone has been demonstrated (Furchgott and Zawadzki, 1980) and it is now known that the action of a variety of vasodilators and vasoconstrictors is dependent upon an intact endothelium (Angus and Cocks, 1989). However, the mechanisms underlying the endothelial integrity remain unknown.

Since vascular endothelial growth factor (VEGF), the obligatory survival factor for the endothelial cells (ECs), signals through c-kit receptors (Sakao et al., 2007), the survival of the ECs in the blood vessels might be linked to the kit signalling in one of the neighbouring cells.

The first question raised in the present study, therefore, was whether there is a subset of cells in the umbilical vein wall close to the endothelium involved in kit signalization. The second question was whether ultrastructural differences can be revealed in the umbilical vein walls of full-term and pre-term neonates and whether and to what extent these differences may relate to the endothelial integrity. Accordingly, umbilical veins were subjected to conventional electron microscopy, immunoelectron microscopy and immunohistochemistry immediately after delivery. On the basis of the gestational age at delivery, the infants were divided into fullterm and pre-term groups.

\section{Materials and methods}

\section{Collection and preparation of tissues}

Human umbilical cords from full-term $(n=14$, range 37 41 weeks, mean $39.0 \pm 1$ weeks $)$ and pre-term $(n=8$, range 31-36 weeks, mean $33.5 \pm 1$ weeks) vaginal deliveries were obtained from the Department of Obstetrics and Gynaecology at the University of Szeged. Two of the eight pre-term neonates were diagnosed with restricted intrauterine growth, whereas no indication of a disturbed UmbBF was noted in the other newborns.

The human study was approved in advance by the University Medical Ethical Committee and was performed in accor- 
dance with the ethical standards laid down in the 1964 Declaration of Helsinki. Thick segments $(2-3 \mathrm{~cm})$ of the cords were cut $10 \mathrm{~cm}$ distally to the placenta and were intensively washed in phosphate buffer $(\mathrm{PB} ; 0.05 \mathrm{~m}, \mathrm{pH} 7.4)$ at $4^{\circ} \mathrm{C}$. When the cord tissues were visibly bloodless, selected segments were immersed in fixative containing 2\% paraformaldehyde and 2\% glutaraldehyde in PB for electron microscopy, or $4 \%$ paraformaldehyde for light microscopy, and stored overnight at $4^{\circ} \mathrm{C}$. The following day, the umbilical veins were washed in $\mathrm{PB}$ dissected free of surrounding tissue and cut transversely to produce strips $3-5 \mathrm{~mm}$ in length. The tissue strips were then processed for electron microscopy or for paraffin sectioning.

\section{Post-embedding immunocytochemistry}

Whole mounts fixed for electron microscopy were embedded in Epon and sections $70 \mathrm{~nm}$ in thickness were transferred onto nickel grids coated with Formvar film. For immunogold staining, the grids were first placed onto droplets of TBS containing $1 \%$ BSA (TBS-BSA) for $30 \mathrm{~min}$, transferred to droplets containing anti-c-kit (DAKO, Glostrup, Denmark polyclonal) diluted in TBS-BSA (final dilution 1:250), and incubated in a humid chamber overnight. After washes with TBS, the grids were placed on droplets of gold-labelled secondary antibody (5 $\mathrm{nm}$ gold, Sigma, final dilution 1:50) diluted in TBS-BSA containing $0.5 \%$ Tween 20 , for $2 \mathrm{~h}$, then washed. Staining for contrast was in a saturated aqueous solution of uranyl acetate for $5 \mathrm{~min}$, followed by lead citrate for $2 \mathrm{~min}$. Ultrathin sections were examined in a Philips CM10 electron microscope equipped with a MegaView II camera.

\section{Quantitative methods}

Via the morphological characteristics of the apoptosis-necrosis cell death continuum (Rost-Roszkowska, 2008), a threegrade and a two-grade scale were applied to classify the degree of the degeneration of the SMCs and the ECs, respectively. The proportions of normal, dying and dead SMCs were determined, using 100 cells per vein for the scoring. The proportions of normal and dying ECs were determined by using 100 ECs per vein for the scoring.

\section{Bax immunocytochemistry}

All incubations were carried out at room temperature. Eight$\mu$ m-thick paraffin sections were treated with $0.1 \% \mathrm{H}_{2} \mathrm{O}_{2}$ in $\mathrm{PB}$ for $20 \mathrm{~min}$ to eliminate endogenous peroxidase activity, and then blocked in $0.05 \mathrm{~m}$ PB containing $0.1 \%$ BSA and $10 \%$ normal goat serum for $30 \mathrm{~min}$. Sections were incubated with anti-Bax (BD Biosciences, Erembodegem, Belgium; final dilution 1:500) as primary antibody. After washing in $0.05 \mathrm{M}$ $\mathrm{PB}$, sections were incubated with biotinylated anti-rabbit IgG (Amersham, final dilution 1:100), for $6 \mathrm{~h}$, followed by an overnight incubation with streptavidin-biotinylated horseradish peroxidase (Amersham, Buckinghamshire, UK; final dilution 1:100). Peroxidase activity was revealed by means of DAB as chromogen. Sections were then mounted in glycerol-PB, observed and photographed via a Leica DMLB light microscope equipped with a Polaroid digital camera.

\section{Controls}

Throughout the present study, control preparations were performed by omission of either the primary or the secondary antibody, when no immunolabelling was detected.

\section{Statistical analyses}

Statistical analysis was performed by using one-way ANOvA with the Newman-Keuls post hoc test for multiple group comparisons with GraphPad Prism 4 software (San Diego, CA, USA). A probability of $P<0.05$ was set as the level of significance in all analyses. Data were expressed as means $\pm \mathrm{SD}$.

\section{Results}

Umbilical veins from full-term and pre-term neonates were examined by means of electron microscopy and immunohistochemistry. In the full-term neonates, the lumen of the vessels was lined with a continuous sheet of ECs, with the electron-dense lamina elastica interna (LEI), lying below (Fig. 1A). Morphological indications of apoptosis were frequently seen in the ECs, but the membrane integrity

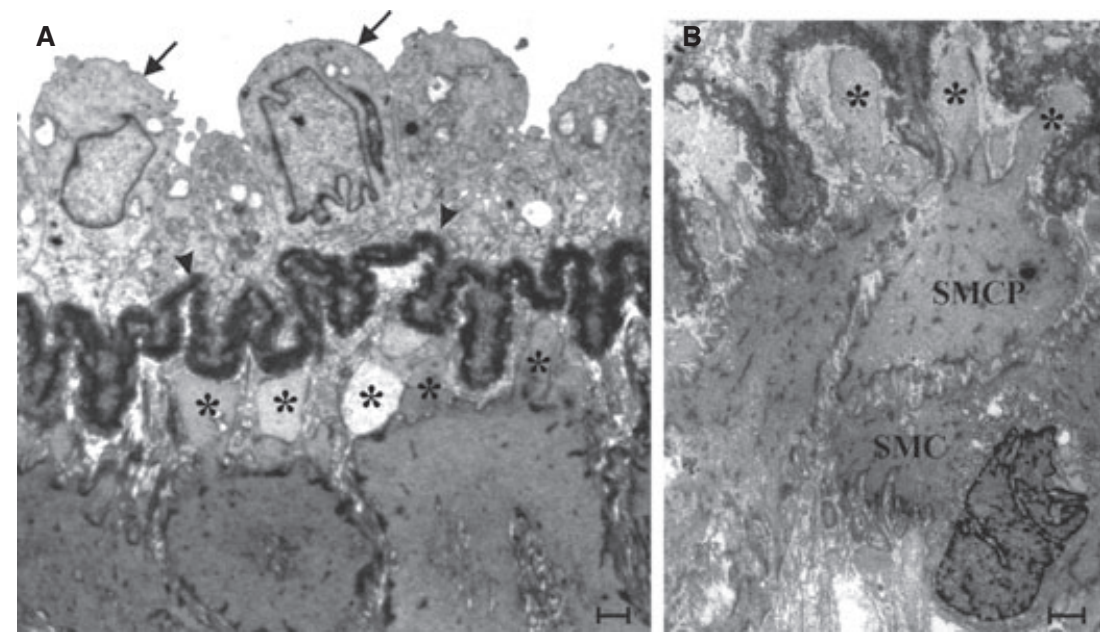

Fig. 1. Electron micrograph of the wall of a human umbilical vein of a full-term neonate. The lumen of the vessel is lined with endothelial cells (A, arrows), below which lies the electron-dense lamina elastica interna (arrowheads). The smooth muscle cells with processes

(SMCPs) in the upper layer of the tunica media (B, SMCP) exhibit electron-lucent foot-like processes protruding to the lamina elastica interna (asterisks). However, the cell body of the SMCPs is structurally similar to that of the neighbouring smooth muscle cells (SMC). Scale bars: $1 \mu \mathrm{m}$. 
was always maintained (Fig. 1). The tunica media consisted of a densely packed smooth muscle layer (Fig. 1). An abundance of collagen-filled spaces was visible at the surface of the vessels continuous with Wharton's jelly, which appears to serve the function of adventitia here (not shown). The cells in the upper layer of the tunica media had an irregular shape and exhibited numerous, long, foot-like processes that were closely associated with the LEI without intervening basal lamina (Fig. 1). The interior of these footlike processes consisted of electron-lucent, homogeneous cytoplasm, while the cell bodies displayed more myoid features, with more electron-dense cytoplasm, scattered caveolae, dense bodies and a continuous, thin basal lamina (not shown).

After post-embedding immunoelectron microscopy, using ckit antibody, these cells with irregular shapes and foot-like processes from the tunica media selectively demonstrated c-kit immunoreactivity, while the remainder of the SMCs and the ECs were negative for c-kit (Fig. 2). Most of the c-kit immunoreactivity proved to be scattered in the cell processes, close to the LEI (Fig. 2). A considerable number of the SMCs with processes (SMCPs) presented features of severe necrosis, e.g. swelling of the cell, with disruption of the organelles and rupture of the plasma membrane in the umbilical vein of both the full-term (Fig. 1) and the pre-term neonates (Fig. 3);

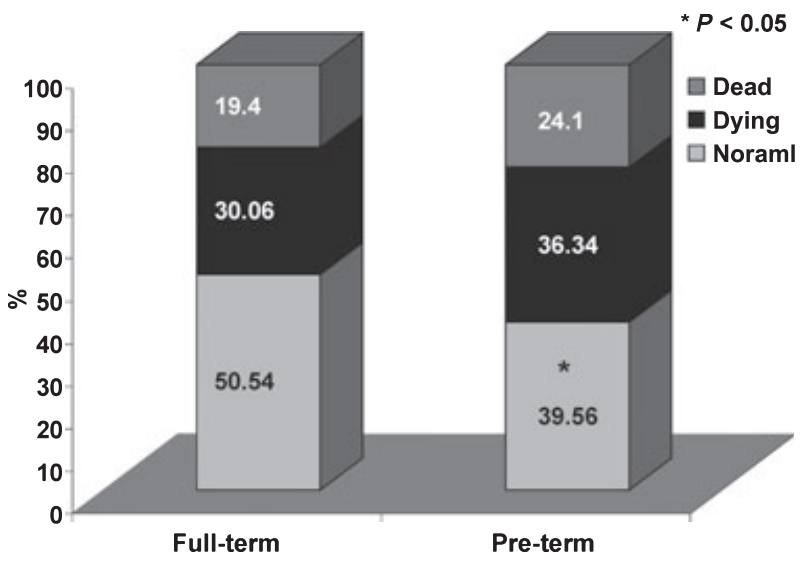

Fig. 4. Histogram showing the proportions of normal, dying and dead processes of the smooth muscle cells with processes of the umbilical vein in full-term and pre-term neonates. The proportion of the normal processes was significantly lower $(P<0.05)$ in the pre-term neonates compared to the full-term neonates. The proportions of dying and dead processes were considerably higher in the veins of the pre-term ones.

however, the structural signs of necrosis in the umbilical vein were more pronounced in the pre-term infants. The severity of necrosis was related to the length of gestation at the time of
Fig. 2. High-power electron micrograph of the wall of a human umbilical vein of a full-term neonate after post-embeddingimmunoelectron microscopy, using the anti-c-kit primary antibody. SMCP, smooth muscle cells with processes. Scale bars: $200 \mathrm{~nm}$.

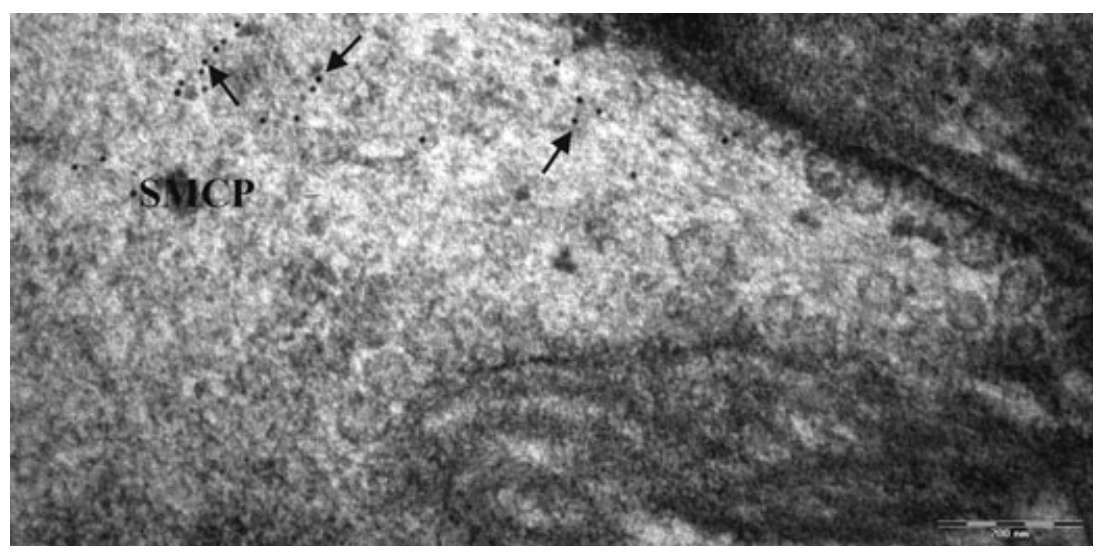

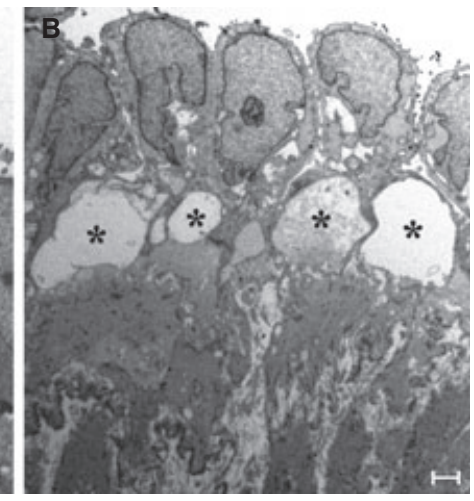

Fig. 3. Electron micrographs of the wall of human umbilical veins of two pre-term neonates (A week 31 of gestation, B week 36 of gestation). The severity of necrosis proved to be related to the length of gestation at the time of delivery. A considerable number of the smooth muscle cells with processes (SMCPs) displayed features of severe necrosis (asterisks), e.g. swelling of the cell with disruption of the organelles and rupture of the plasma membrane in both umbilical veins. At week 31 of gestation (A), not only the advanced necrotic processes in the SMCPs were obvious, but also intense apoptotic processes of the endothelial cells, characterized by shrinkage of the cells, membrane blebbing, intracellular vacuoles and chromatin condensation (arrows). Scale bars: $1 \mu \mathrm{m}$. 
delivery (Fig. 3). The earliest delivery involved in the present investigation occurred in week 31 of gestation, when not only the advanced necrotic processes in the SMCPs, but also intense apoptotic processes of the ECs were obvious, characterized by shrinkage of the cells, membrane blebbing, intracellular vacuoles and chromatin condensation (Fig. 3). As a result of the considerable shrinkage of the ECs, the inter-cellular junctions between the apoptotic cells and the adjacent cells disappeared and the endothelium became leaky. Quantitative analysis indicated (Fig. 4) that the proportion of SMCPs with a normal ultrastructure was significantly lower $(P<0.05)$ in the pre-term veins $(39.6 \pm 6.4 \%)$ than in the full-term veins $(50.5 \pm 15.4 \%)$, and as a consequence the proportions of the dying and the dead processes were higher in the pre-term neonates $(36.3 \pm 5.3 \%$ and $24.1 \pm 5.3 \%)$ than in the full term ones $(30.1 \pm 10.5 \%$ and $19.4 \pm 12.8 \%)$. Similar quan-

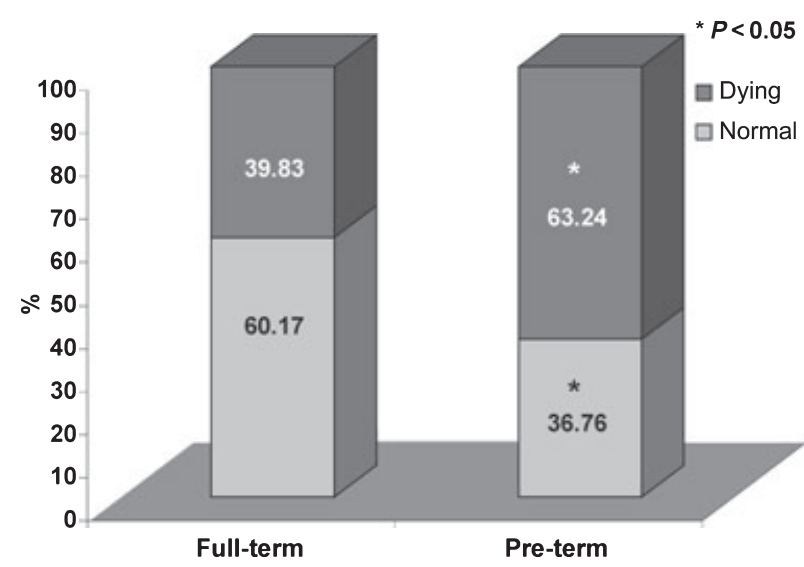

Fig. 5. Histogram showing the proportions of normal and dead endothelial cells (ECs) of the umbilical vein in the full-term and the pre-term neonates. The proportion of the normal ECs was significantly lower $(P<0.05)$ for the pre-term neonates relative to the full-term ones. titative features were revealed, when the ECs were considered (Fig. 5). After full-term delivery, $39.82 \pm 21.60 \%$ of the ECs exhibited morphological signs of apoptosis: intracellular vacuoles, membrane blebbing and chromatin condensation. After pre-term delivery, the proportion of dying ECs was significantly higher $(63.2 \pm 17.7 \%, P<0.05)$.

After full-term delivery, on the application immunocytochemistry with the proapoptotic marker Bax, the intensity of the staining of the endothelium was hardly above the background level (Fig. 6A), whereas strong and highly selective staining of the ECs was seen in the umbilical vein of the preterm infants (Fig. 6B).

\section{Discussion}

The previously undetected SMCPs were characterized in this study by electron microscopy and immunohistochemistry in the upper layer of the tunica media in the wall of the umbilical vein of both the pre-term and the full-term neonates. These cells can be distinguished from regular SMCs both morphologically and immunohistochemically. The most obvious features of these cells are the presence of long, foot-like processes and the c-kit immunoreactivity. Since VEGF, the obligatory survival factor for the ECs, signals through c-kit receptors (Sakao et al., 2007) we presume that the SMCPs through the kit signalling help to maintain the integrity of the endothelium through the whole length of pregnancy. Since the umbilical vessels almost completely devoid of nerves (Fox and Khong, 1990; Benirschke and Kaufmann, 2000; Marzioni et al., 2004), the vasodilators and vasoconstrictors produced by the intact endothelium (Angus and Cocks, 1989) are determinative in the control of vascular tone, and in the control the diameter of the veins, with which the UmbBF is strongly correlated (Link et al., 2007).

Our quantitative results revealed that the numbers of necrotizing SMCPs and apoptotic ECs were significantly higher in the pre-term neonates than in the full-term ones.

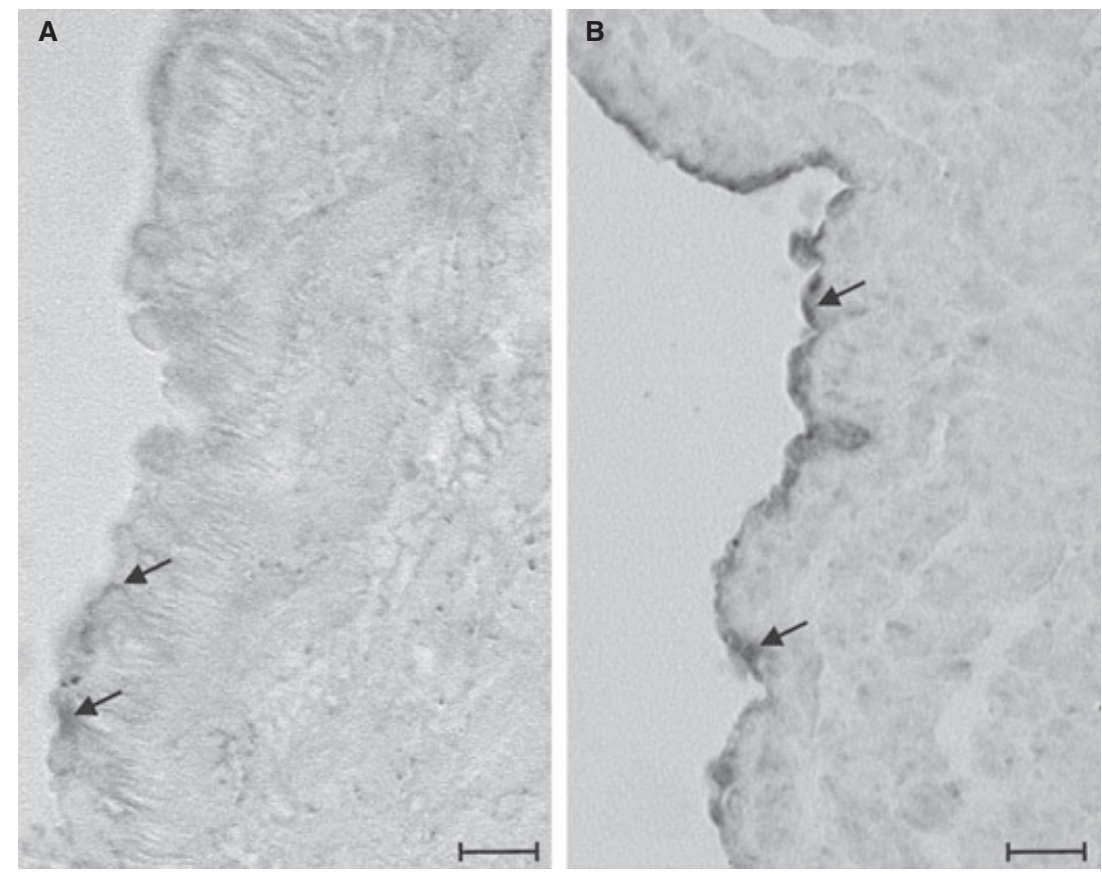

Fig. 6. Photomicrographs of the full-term (A) and the pre-term (B) umbilical vein after Bax immunohistochemistry. After full-term delivery immunocytochemistry with the proapoptotic marker Bax, the intensity of the staining of the endothelium was hardly above the background level (A, arrows), while strong and highly selective staining of the endothelial cells was seen in the umbilical vein of the pre-term infants (B, arrows). Scale bars: $30 \mu \mathrm{m}$ 
These quantitative data and also the selective high expression of Bax proapoptotic gene product in the ECs of the umbilical vein of the pre-term infants indicate that early deliveries are accompanied by the early death of SMCPs and ECs in the umbilical vein. This strengthens our hypothesis that, while the naturally occurring death of SMCPs and ECs plays an essential role in neonatal vascular remodelling during normal embryonic development, a pathological uterine environment may speed up this predetermined death programme. The early regression of the SMCPs and as a consequence the early death of the ECs would result in a reduced $\mathrm{UmbBF}$ and an early termination of pregnancy (Kim et al., 2000).

\section{References}

Angus, J. A., and T. M. Cocks, 1989: Endothelium-derived relaxing factor. Pharmacol. Ther. 41, 303-352.

Benirschke, K., and P. Kaufmann, 2000: The Pathology of the Human Placenta. New York: Springer.

Dawes, G. S., 1969: The placenta and foetal growth. In: Foetal and Neonatal Physiology. (G.S. Dawes, ed.). Year Book Medical Publishers, Chicago, pp. 67.

Di Naro, E., L. Raio, F. Ghezzi, M. Franchi, F. Romano, and D. V. Addario, 2002: Longitudinal umbilical vein blood flow changes in normal and growth-retarded fetuses. Acta Obstet. Gynecol. Scand. 81, 527-533.

Ferrazzi, E., S. Rigano, M. Bozzo, M. Bellotti, N. Giovannini, H. Galan, and F. C. Battaglia, 2000: Umbilical vein blood flow in growth-restricted fetuses. Ultrasound Obstet. Gynecol. 16, 432-438.

Fox, S. B., and T. Y. Khong, 1990: Lack of innervation of human umbilical innervation. An immunohistological and histochemical study. Placenta 11, 59-62.
Furchgott, R. F., and J. V. Zawadzki, 1980: The obligatory role of endothelial cells in the relaxation of arterial smooth muscle by acetylcholine. Nature 288, 373-376.

Kim, H. S., K. K. Hwang, J. W. Seo, S. Y. Kim, B. H. Oh, M. M. Lee, and Y. B. Park, 2000: Apoptosis and regulation of Bax and Bcl-X proteins during human neonatal vascular remodeling. Arterioscler. Thromb. Vasc. Biol. 20, 957-963.

Link, G., K. E. Clark, and U. Lang, 2007: Umbilical blood flow during pregnancy: evidence for decreasing placental perfusion. Am. J. Obstet. Gynecol. 196, e1-e7.

Marzioni, D., L. Tamagnone, L. Capparuccia, C. Marchini, A. Amici, T. Todros, P. Bischof, S. Neidhart, G. Grenningloh, and M. Castellucci, 2004: Restricted innervation of uterus and placenta during pregnancy: evidence for a role of the repelling signal semaphorin 3A. Dev. Dyn. 231, 839-848.

Reilly, R. D., and P. T. Russell, 1977: Neurohistochemical evidence supporting an absence of adrenergic and cholinergic innervation in the human placenta and umbilical cord. Anat. Rec. 188, 277286.

Rost-Roszkowska, M. M., 2008: Degeneration of the midgut epithelium in Allacma fusca L. (Insecta, Collembola, Symphypleona): apoptosis and necrosis. Zoolog. Sci. 25, 753-759.

Sakao, S., L. Taraseviciene-Stewart, C. D. Cool, Y. Tada, Y. Kasahara, K. Kurosu, N. Tanabe, Y. Takiguchi, K. Tatsumi, T. Kuriyama, and N. F. Voelkel, 2007: VEGF-R blockade causes endothelial cell apoptosis, expansion of surviving CD34+ precursor cells and transdifferentiation to smooth muscle-like and neuronallike cells. FASEB J. 21, 3640-3652.

Sgambati, E., M. Marini, G. D. Zappoli Thyrion, E. Parretti, G. Mello, C. Orlando, L. Simi, C. Tricarico, G. Gheri, and E. Brizzi, 2004: VEGF expression in the placenta from pregnancies complicated by hypertensive disorders. BJOG 111, 564-570. 